\title{
The Emergence and Evolution of the Universe
}

\author{
Lev Z. Vilenchik* ${ }^{\circledR}$, Maria Vilenchik \\ Felicitex Therapeutics, Natick, USA \\ Email: *lev.vilenchik@gmail.com, mvilenchik@gmail.com
}

How to cite this paper: Vilenchik, L.Z. and Vilenchik, M. (2019) The Emergence and Evolution of the Universe. Journal of High Energy Physics, Gravitation and Cosmology, 5, 884-898. https://doi.org/10.4236/jhepgc.2019.53044

Received: May 21, 2019

Accepted: July 12, 2019

Published: July 15, 2019

Copyright $\odot 2019$ by author(s) and Scientific Research Publishing Inc. This work is licensed under the Creative Commons Attribution International License (CC BY 4.0).

http://creativecommons.org/licenses/by/4.0/ (c) (i) Open Access

\begin{abstract}
The cosmological picture of the World is complemented by the inclusion of virtual objects, phenomenon of the mass defect, entropic interaction, and bifurcations. During the period before the emergence of our Universe, space is considered as an infinite homogeneous multifold of virtual point objects with a certain energy and are capable of transitioning into a real state of certain masses, electrical charges, and sizes once at irreversible local fluctuations of density and energy. The existence of such virtual point objects is explained on the basis of the mass defect phenomenon known in nuclear physics and the fact that resultant sum of all forces acting on the object is equal to zero at the homogeneous space. It is shown that the entropic changes caused by irreversible fluctuations should have led to bifurcation and produce the "Big Bang" event with the emergence and subsequent evolution of our Universe. The probability of the set of bifurcations and consequent "Big Bang" events, the origin of the corresponding Universes, the time of their occurring, and initial localization is discussed. The possibility of the transition of virtual objects into a real state is proved using the Principle of Least Action and Le Chatelier's Principle. It is shown that this is a first-order phase transition which is accompanied by radiation of energy, violation of symmetry, the appearance of mass, electrical charge, motion, and other attributes of real state objects. The "negative" and "standard" gravity, the "negative pressure", the expansion of our Universe and its "inflationary" period are explained as realizations of the entropic interaction between objects. The "Corpuscular-Wave and the Corpuscular-Field dualisms", being a manifestation of the Complementarity and the Equivalence Principles, are used for the explanation of the evolution of the Universe. The Anthropic Principle is discussed.
\end{abstract}

\section{Keywords}

Entropy, Evolution, Fluctuations, Bifurcation, Gravity, Mass Defect, Entropy Interaction, Virtual Objects 


\section{Introduction}

The origin of the Universe and the study of global phenomena occurring in it are described in the subsection of physics called cosmology. According to cosmology ideas [1]-[7], "Big Bang" event originated our Universe and it is possible that there were a lot of such events or explosions in space which initiated the creation of other Universes. As a result of one, our Universe was created and its development is explained by the cosmological "Inflation Theory" in its numerous variations [8] [9] [10] [11]. Few objections to this theory are described below: the emergence of the matter from nothing and ignorance of entropic changes in the process of the emergence and evolution of the Universe. The given article makes an attempt to address these objections by supplementing this theory with consideration of virtual objects in the original space before the "Big Bang" and utilizing entropic interaction while accounting for bifurcation phenomenon.

\section{The Concept of Entropic Interaction}

The concept of entropic interaction is usually used in the subjunctive mood. For example, "macromolecular units as if entropically repel each other at close distances and entropically attract at large distances" [12] [13]. The modern science considers, the entropic interaction (as sighted in [14] [15] [16] [17] [18]) as the mutual influence by open thermodynamic systems on each other via exchange of information about their corresponding states, coordinating their entropy changes, and mutually striving to transition to a more probable state. Entropic interaction is considered a quintessential physical interaction, which is realized through well-known fundamental interactions (gravitational, electromagnetic, nuclear strong and weak) within processes that occur throughout the Universe, including the Solar system, our planet, plants, and living organisms. Fundamental interactions are considered as subsidiaries of the entropic interaction.

The entropic interaction is not a consequence of the existence of some entropic charge and its accompanying field. It cannot be stated that the entropic interaction spreads through space "from one point to another" and transfers the energy. It merely reflects a certain "order", "structure", and "state" of space and the physical systems that are within it determining the energy, behavior, and evolution of these systems and the entire space as a whole. Entropic interaction leads to the breaking of symmetry, changes of free energy, as well as entropy and other characteristics of a physical system. Under its influence, the change of entropy in any part of a thermodynamic system becomes a simultaneous change of the entropy of the entire system. That is the entropic interaction is a longrange action [16].

It is useful to remember that an approach with long-range action was used in the Landau Theory [19] for the description of the second-order phase transition utilizing the self-consistent field. According to that theory, the change of symmetry in any part of a system results in the change instantly known to all 
parts of the system.

The similar approach was used by Hartree-Fock method [20] in quantum mechanics to solve the Schrodinger equation by reducing the multiparticle problem to a one-particle problem under the assumption that each particle moves in some homogenized self-consistent field created by all the other particles of the system. At this consideration, any change of the state of just one particle immediately correlates with corresponding changes of states of all particles of the system. In other words, a long-range action was used here to transfer the corresponding physical information.

Another example of the long-range action in quantum mechanics is the quantum entanglement [21] [22] [23]. This is considered usually as a consequence of quantum superposition and is a physical phenomenon that occurs when pairs or groups of particles are generated or interact in such ways that the quantum state of each particle cannot be described independently of the others, even when the particles are separated by a large distance. Instead, a quantum state must be described for the system as a whole.

All of these examples represent the realization of the entropic interaction between different parts of the corresponding thermodynamic system.

All systems being in non-equilibrium state began to evolve. During evolution, the entropy is increasing that leads systems to a more chaotic state. However, it is worth mentioning that entropy increases only as a result of irreversible processes, that is, those that cannot pass in the opposite direction through all the same intermediate states. All real processes are irreversible due to the interaction of the objects participating in them. Examples include diffusion, thermal conductivity, radioactive decay, and many others.

Under non-equilibrium conditions when the free energy is decreasing, a change of the entropy can produce not only degradation but order, organization and ultimately life. That is the non-equilibrium state can be a source of order which is generated as "order out of chaos" [24] [25].

Using the entropic interaction, all objects in Nature (including homo sapiens) exert an influence on one another, regardless of the distance between them, which can be considered as an extended interpretation of the Mach Principle [6] [26] [27] [28], and entropic interaction as the quintessence of the Universe, determining its evolution. (According to I. Prigogine, translation from the Greek language, “entropy” ( $\left.\dot{v} \nu \rho \circ \pi^{\prime} \alpha\right)$ means “evolution”). It is interesting that as early as in the 17th century, an eminent mathematician and physicist, Leibniz, stated the following inconsonant with the Mach principle: "The Universe is an interconnected whole".

\section{Observation of Material Objects and Mass Defect}

Before proceeding to the description of the origin and development of our Universe, let's recall that the mass of an object is a measure of its gravity, inertia and energy [29], which demonstrates itself only when an object is acted upon by any force. If there is no such effect, or the geometric (resultant) sum of all forces 
acting on the object is zero, then the mass of the object would not be manifested in any way. The same can be stated about other characteristics of material objects such as electric charge, "visibility", etc. An electric charge "manifests" itself once it is acted upon by another electric charge. The object becomes visible when it either reflects or emits the light. Objects without an electrical charge cannot radiate, absorb or reflect the electromagnetic radiation. Dark matter [30] can be as an example of that. Niels Bohr formulated these phenomena as follows: "There is no reality that does not depend on the method of its observation".

The state of weightlessness is an example when the resultant force acting on an object is equal to zero. Any object demonstrates this when it either moves by inertia away from the other massive objects or falls freely on bodies having mass or when it moves along a curvilinear trajectory with appropriate speed, such as the orbit of a man-made Earth satellite. The gravitational attraction of the Earth is compensated by the force caused by the acceleration of free fall $g$, and in orbit by the centrifugal force $m v^{2} / r$. These forces realize the entropic interaction between the Earth and the corresponding object and manifest the entropic repulsion. In each case, the gravitational attraction of objects is compensated by a corresponding decrease in their entropy, leaving unchanged the free energy $G$ of a given thermodynamic system. As a result, the behavior of objects does not depend on their mass.

Another example is the chromatography of macromolecules performed under critical conditions, discovered by the author and his colleagues in 1974 [31]. During the chromatographic process, substances are separated according to their molecular size, molecular weight, and interaction with the surface of porous sorbent particles, along which they are moved by the solvent flow. When the macromolecule reaches the available pore that correlates to its size, it lingers at this pore for some time and lags behind the flow. During its stay in the pore, the entropy of the molecule is reduced, since the macromolecule cannot realize all its conformations. At the same time, adsorption interaction with the surface takes place inside the pores, decreasing enthalpy of macromolecules. Under critical conditions, enthalpic and entropic interactions completely compensate each other and, as a result, all molecules, regardless of their molecular weight and size move at the same velocity. In this case, the resultant force acting on the macromolecules is zero and masses and sizes of the macromolecules do not appear. The role of forces is played by the force of the adsorption attraction to the surface of pores and the elastic force of the macromolecular chain that realizes the entropic repulsion from the surface.

It is well established in nuclear physics that the mass of an atomic nucleus is always less than the total mass of its constituent nucleons (especially for sufficiently large nuclei with a number of nucleons greater than 50). This mass defect is explained by mass "consumption" for the binding energy of nucleons in the nucleus of an atom. Moreover, the mass defect is reversible: the mass spent on the binding energy does not disappear forever, and if the nucleus could be 
"disassembled" into the nucleons entering it, then each of them would have its original mass.

A similar mass defect (according to theoretical calculations) is demonstrated (for example) by the three quarks ("Up", "Down", and "Charm") that make a proton.

As a distance between either objects or parts of the same object decreases, their gravitational interaction and binding energy increase, while enthalpy, and entropy decrease. (Free energy at the same time or decreases, or may remain unchanged). The mass defect becomes greater and one can imagine the extreme case when the mass defect equal to the mass of the object and its size has become a point-like one. An example is a photon having a zero mass at rest and in the theory of electroweak interaction [32] considered as a superposition of two gauge bosons having significant masses.

The reversibility of the mass defect states that such objects continue to be present in space, but being in a virtual state, i.e. a state in which the mass defect is equal to the mass, the resultant sum of all forces acting on the object is zero and the object is not observed but can manifest itself when these conditions are not satisfied. Here it is appropriate to quote Lucretius, Greek poet and philosopher, who was the first to suggest that "there are objects that we cannot see".

\section{World Space before the "Big Bang"}

The structure of the space prior to "Big Bang" can be described as a multifold of virtual point objects, for which mass defect is equal to their masses. Space was infinite, homogeneous, isotropic, and possessed both energy and maximum possible entropy. The absence of real objects makes it possible to speak about a physical vacuum of this space, consisting of very dense structureless ("chaotic") matter, which was in a virtual state. Beside of insignificant reversible fluctuations of the density and the energy of this matter, no processes occurred in space. The lack of any processes leading to the evolution of space means lack of counting of time, i.e. absolute timeline did not exist. (Let us note, the time is a way to evaluate and compare the speeds of processes occurring in Nature [26]. The absence of processes, which lead to changes of the state of a particular system, means that either there is no time flow in the system or the flow stopped.)

However, as a result of some local quantum fluctuations which can be very strong, the vacuum density and energy in various small regions of this space could change abruptly above a certain critical value. At this turning point, called bifurcation point [24] [25], it is impossible to predict the direction of the further development: would the state of the system remains chaotic or would it go to a certain level of orderliness and structure of the space that existed before, would not be maintained and therefore would collapse. There could be many such areas and bifurcations. These bifurcations occurred randomly and continually and did not coordinate with each other. As a result of them, the entropy of the space (and binding energy for virtual objects) could decrease and free energy 
increased. State of the space became unstable and definite processes, that led to an evolution of the space, were initiated.

\section{Evolution of the World Space}

For the evolution of the World space, certain processes should occur and the count of time should begin in every disturbing local region where the bifurcation has led to an unstable state of space. A mass defect for virtual objects ceased to be equal to their masses because of a decrease of the binding energy. Objects transferred from a virtual state to a real one like at a first-order phase transition of a matter (similar to freezing of any liquid). Because of entropy decrease in these local regions, space became heterogeneous. The sum of forces caused by the interaction of objects in the heterogeneous space appeared to be non-zero. Selected directions, along which forces acted, appeared in the space. Masses of these objects began to "manifest" themselves and their sizes expand. Objects transferred to a real state as elementary particles (including dark matter that hasn't an electrical charge) and perhaps, more complex entities and formed a thermodynamic system in every disturbing local region. Evolution of these systems had not been a completely stochastic process but was rather developing towards the direction where free energy of space would decrease. The tendency to decrease the free energy stimulated the combining of these particles into atoms, molecules and macroscopic objects such as planets, stars, and galaxies. The irreversibility of such process generates definite levels of the space organization that means the evolution is developing in the direction from simple to complex (including the organic world). It is possible to say: Matter becomes "active": it generates irreversible processes, and irreversible processes organize matter. As it gets older, the universe is becoming a more subtle organization. Over time, the level of organization of the universe steadily increases [24] [25]. During a short period of time, when the objects in each local region started to move, the probability of their contacts and various combinations was very high as the objects were still close enough to each other and the energy of their mutual attraction was significantly larger than the decrease of the entropy at the combinations. At the same time, there was a probability that the entropic interaction between objects turned on processes that stimulated a divergence of local regions and caused the entropic repulsion of objects. The systems of these objects were evolving. Their symmetry was destroyed. That means that some conservation laws inherent in it were violated in accordance with the Noether Theorem [33]. This evolution turned out to be irreversible, due to the interaction of objects [34].

\section{Using the Principle of Least Action to Describe the Origin of the Universe}

The tendency to such evolution is well known in thermodynamics and can be easily derived from the Principle of Least Action [35] [36] [37] [38]. According 
to this Principle, all changes in Nature should be such that some of its quantitative characteristics (usually associated with energy) remain minimal. Moreover, the evolution of an object should be such that its' functional of action $S$ would remain unchanged.

$$
\begin{gathered}
S=\int_{t_{1}}^{t_{2}} L\left(q_{i}(t), \frac{\partial q_{i}}{\partial t}\right) \mathrm{d} t \\
\delta S=0,
\end{gathered}
$$

where $L$ is the Lagrange function of the object, which is not being explicit a function of time and depends on any number $n$ independent values $q_{i}$, which, together with their time derivatives, fully characterize the state of the object with $n$ degrees of freedom $(i=1,2, \cdots, n)$ and are called generalized coordinates and generalized velocities, respectively.

In thermodynamics, Cartesian coordinates $x_{i}$, heat $Q$, temperature $T$ and entropy $s$ can be used as generalized coordinates. Taking them into account leads to the following action functional for a thermodynamic system in the one-dimensional case:

$$
S=\int_{t_{1}}^{t_{2}} L\left(x, x_{t}, S, s_{t}, Q, Q_{t}, T, T_{t}\right) \mathrm{d} t
$$

Since the thermodynamic characteristics of the $s, Q, T$, acting as generalized coordinates, are not arbitrary (independent) functions, it is necessary to consider the variation of the action functional (Equation (3)) together with the relevant condition's connection, as is done in variational problems on the conditional extremum [39]. In this case, the requirement Equation (2) for the stationarity of the action $S$ leads to the Equation (4):

$$
\begin{aligned}
\delta S= & \int_{t_{1}}^{t_{2}}\left[\delta x \partial L / \partial x+\delta x_{t} \partial L / \partial x_{t}+\delta s \partial L / \partial s+\delta s_{t} \partial L / \partial s_{t}\right. \\
& \left.+\delta Q \partial L / \partial Q+\delta Q_{t} \partial L / \partial Q_{t}+\delta T \partial L / \partial T+\delta T_{t} \partial L / \partial T_{t}\right] \mathrm{d} t=0
\end{aligned}
$$

We are interested in the variation of the action functional $S$ on the conditional extremum on a small ("inflationary") time interval (the order of the Planck time $\left.t_{p}=5.39 \times 10^{-44} \mathrm{sec}\right)$. Then the integral in Equation (4) vanishes when the integrand is zero:

$$
\begin{aligned}
& \delta x \partial L / \partial x+\delta x_{t} \partial L / \partial x_{t}+\delta s \partial L / \partial s+\delta s_{t} \partial L / \partial s_{t} \\
& +\delta Q \partial L / \partial Q+\delta Q_{t} \partial L / \partial Q_{t}+\delta T \partial L / \partial T+\delta T_{t} \partial L / \partial T_{t}=0
\end{aligned}
$$

As the Lagrange function $L$ for such a system, it is essential to use the expression for the Gibbs free energy $G$, the enthalpy part of which includes the energy associated with the resting mass of the object $m_{0}$, its kinetic energy $m_{0} c^{2} / \sqrt{\left(1-x_{t}^{2} / c^{2}\right)}$, the amount of heat $Q$ and the energy $U$ of interaction of the object with a certain force field $F$ included in the system $(U=-F x)$. The entropy part of $G$ is $(-s T)$.

Accordingly, the Lagrange function is described as the following:

$$
L=G=F x+m_{0} c^{2} / \sqrt{\left(1-x_{t}^{2} / c^{2}\right)}+Q-s T
$$


Substituting the Lagrange function written in the form of the Equation (6) into Equation (5) we obtain

$$
\begin{aligned}
\delta s=\{ & F \delta x-\delta x\left[m_{0} x_{t t} / \sqrt{\left(1-x_{t}^{2} / c^{2}\right)^{3}}\right. \\
& \left.\left.+m_{0} x_{t t}\left(3 x_{t}^{2} / c^{2}\right) /\left(1-x_{t}^{2} / c^{2}\right)^{5 / 2}\right]+\delta Q-s \delta T\right\} / T
\end{aligned}
$$

On the basis of Equation (7), it can be concluded that choosing a Lagrangian in accordance with Equation (6), the reduction of entropy $\delta s$ for each small region (commensurate with the Planck length $l_{p}=1.616 \times 10^{-35} \mathrm{~m}$, where the vacuum density changed in an irreversible way) is implemented by emergence of objects with a mass $m=m_{0} / \sqrt{\left(1-x_{t}^{2} / c^{2}\right)}$, moving with speed $x_{t}$ at acceleration $x_{t t}$ and having a value of $m_{0}$ at rest. The more the entropy decreases, the larger the velocity of the objects. The presence of a force field (described as $F \delta x$ in Equation (7)) increases the entropy if this field is potential and the force $F$ acts as a repulsion from the source of the field. If this force acts as an attraction, i.e. the field, for example, is the gravitational field, the term $F \delta x$ in Equation (7) is negative and leads to a decrease in entropy. A decrease in entropy in Equation (7) also leads to the removal of heat from local regions ( $\delta Q<0$ and to a decrease of their temperature $(\delta T<0)$.

The description of the processes occurring in the space filled with a scalar field $\varphi$ leads to similar results, at his perturbation and a corresponding decrease in entropy. In this case, if for the Lagrangian of a single-component scalar field $\varphi$ choose the expression

$$
L=1 / 2(\partial \varphi / \partial x)^{2}-s T+Q-\left(m^{2} c^{2} / 2 h^{2}\right) \varphi^{2}-(\lambda / 4) \varphi^{4},
$$

in which are traditionally used [40] in the Lagrangian difference of kinetic energy $1 / 2(\partial \varphi / \partial x)^{2}$ and potential energy $\left(m^{2} c^{2} / 2 h^{2}\right) \varphi^{2}-(\lambda / 4) \varphi^{4}$ is replaced by the free energy $G$ (including its entropic component $(-s T)$ and the amount of heat in the system $Q$ ). Next, substituting the expression for the Lagrangian (8) into Equation (3) for the action $S$ and requiring execution (2), we obtain from the conditional extremum of the action functional $S$ :

$$
\begin{aligned}
\delta s= & \left\{(\partial \varphi / \partial x)\left[\delta x\left(\partial^{2} \varphi / \partial x^{2}\right)-2\left(m^{2} c^{2} / 2 h^{2}\right) \varphi-\lambda \varphi^{3}\right]\right. \\
& \left.-2\left(m c^{2} / 2 h^{2}\right) \varphi^{2} \partial m / \partial x_{t}+\delta Q-s \delta T\right\} / T
\end{aligned}
$$

where $\lambda$-coupling constant, which determines the interaction with the field objects located therein.

The presence of the positive term $\delta x(\partial \varphi / \partial x)\left(\partial^{2} \varphi / \partial x^{2}\right)$ in the Equation (9) in addition to earlier conclusions states the increase in the size of the region of space with reduced entropy (factor $\delta x$ ), the appearance of a density gradient and field source in it (multipliers $(\partial \varphi / \partial x)$ and $\left.\left(\partial^{2} \varphi / \partial x^{2}\right)\right)$. Those, the use of a scalar field $\varphi$ to describe a process that implements a stepwise decrease in the entropy of space, then its gradual increase, also leads to the earlier made 
conclusion about the expansion of local areas of space, the appearance of heterogeneity and non-isotropy, the violation of symmetry and the appearance of material objects with motion, energy and mass.

It is seen from Equation (9) that the mass of the objects appearing in the local region of space with decreasing entropy $((\delta s<0,-T \delta s>0))$ is larger with the greater decrease of entropy, more heat radiates $(\delta Q>0)$ in this region and the more it is cooled in the process $(\delta T<0,-s \delta T>0)$.

Radiation of heat means that when a transition to a real state occurs, objects acquire electric charges and the corresponding electromagnetic fields take part in their interaction. The appearance of these fields follows not only from the Principle of Least Action but also from the Le Chatelier's Principle [41] which states that Nature is an inertial and thermodynamic system opposes any changes in its state caused by external forces. This is well illustrated by the example of the Dirac equation [42]

$$
\sum_{k=0}^{3} \alpha_{k} \partial \psi(x) / \partial x_{k}+4 \pi i(m c / h) \psi(x)=0,
$$

where $\psi(x)$ is wave function (bispinor), $\alpha_{k}$ are Dirac matrices.

Equation (10) describes the behavior of a free, non-interacting particle. Such a particle is in a stable state that can fluctuate reversibly within the limits of Heisenberg's Uncertainty Principle. However, when a particle passes at the bifurcation point from the virtual to the real state, the symmetry of the Equation (10) breaks down and the extra term $i e \sum_{k=0}^{3} \alpha_{k}\left(\partial \varphi(x) / \partial x_{k}\right) \psi(x)$ appears in there when the wave function rotates at an arbitrary angle $\varphi$ in the "charge space" [42]. This rotation can be written as follows:

$$
\psi(x)=\psi(x) \exp [\operatorname{ie\varphi }(x)],
$$

where $e$ is a charge of an electron.

To eliminate the extra term (Natura "want" to make this, accordingly to Le Chatelier's Principle) and, consequently, to preserve symmetry, at least one more charged particle and the corresponding electromagnetic field must emerge in space (that is, the particle moves from the virtual to the real state). As a result, the Dirac equation for a charged particle in the electromagnetic field takes an invariant form

$$
\sum_{k=0}^{3} \alpha_{k} \partial \psi(x) / \partial x_{k}+4 \pi i(m c / h) \psi(x)=i e \sum_{k=0}^{3} \alpha_{k} A_{k}(x) \psi(x)
$$

where $A_{k}$ is the potential of the electromagnetic field.

This example shows the possibility of the emergence in space of charged material objects not only at the moment of the Big Bang but also some time afterward.

\section{The Evolution of World Space and Our Universe after the "Big Bang"}

It should be noted that after the introduction of the concept of an electromagnetic field into physics by Faraday and Maxwell and then the gravitational field by 
Einstein, it is considered that everything electrically, magnetically, "gravitationally", or otherwise "charged" objects interact through their own fields, each of which represents some kind of continuous medium transmitting "from point to point" the force action of the respective charges on each other. The emergence of quantum mechanics, and then nuclear physics, introduced into the field concept a significant addition. It turned out that all physical fields consist of quanta (photons, mesons, gluons, not yet detected gravitons, and other elementary particles) which transfer field energy between interacting objects. Thus, a "corpuscular-field dualism" appeared: on the one hand, the field is a continuous medium, and on the other, it is the medium consisting of discrete energy quanta. This dualism, unlike the well-known wave-particle duality, is practically not discussed in the scientific literature, although it has been always present in the most direct way.

Both of these dualisms are the implementation of two fundamental principles: the Principle of Complementarity [43] and the Principle of Equivalence [44], which one can try to generalize to a wide range of phenomena as follows: In certain experiments, the same quality (property) of material objects can manifest itself in fundamentally different ways. In mechanics, this is observed in Newton's second law as force and acceleration, and in Newton's third law as action and counteraction. In quantum mechanics and field theory, this manifests itself in interference and scattering phenomena, in relativity theory, in the existence of gravitational and inertial mass.

For a thermodynamic system, this quality is a change in its free energy $\Delta G$, which leads to the evolution of the system and can occur either through entropy changes $\Delta s$, or enthalpy $\Delta H$, or both: $\Delta G=\Delta H-T \Delta s$.

By using the "Corpuscular-Field dualism", we consider how a striving of a perturbed World space into a more probable state could be realized. For that, material objects, which became real, start entropically interacting with each other by emitting energy quanta (photons, mesons, gravitons, etc.) in randomly chosen directions and receive momentums from absorbed quanta emitted by neighboring objects.

Suppose there are two real objects $A$ and $B$ and each of them has certain energy charges, which include the gravitational charge (mass), electric charge, "nuclear", and any other. Such charges emit the corresponding quanta of energy. In the absence of any interaction between objects (when $t \ll t_{p}$ ), this radiation is stochastic, i.e. all its directions are equally probable (as if only one object was in the space). With the emission of quanta, objects receive reactive momentums (recoil pulses) in a direction opposite to the radiation (a kind of Mössbauer effect [45]). Due to the random nature of the radiation, when $t \ll t_{p}$, the recoil momentums received by the objects are added vectorially to a summary total momentum equals zero. However, after the time $t$, that is still less $t_{p}$, but more close to it, the quanta emitted from object $A$ reach object $B$, and the quanta from object $B$ reach object $A$. Some of the quanta, which have a resonant energy [45], 
are absorbed and transmit their momentums $P_{A \rightarrow B}$ and $P_{B \rightarrow A}$ to the objects. Under the influence of these momentums, objects scatter in different directions, as if repelling from each other (termed as "negative gravitation" by Inflation theory), realizing their entropic interaction, which tends to increase the total entropy of objects and to decrease their free energy.

Next, the objects' radiation became non-isotropic due to a specific of their entropic interaction. The tendency to increase entropy and to decrease the free energy, and so to have the same number of quanta of certain energy on both sides of each object (similar to the osmotic pressure), reduces their radiation into the internal region and, accordingly, increases the radiation into the external region by the same amount. The entropic repulsion of the objects is decreasing. Inflation theory termed this effect "appearance of standard gravitation".

However, when the distance between the objects began to be large enough, their energy radiation increases inside the area surrounding the objects and decreases outside of them (because of the same evolutionary tendency). Accordingly, the entropic repulsion will increase as well. This phenomenon is described as "appearance negative pressure" by Inflation theory.

Similarly, the mutual attraction of objects with opposite electric charges and repulsion of objects with charges of the same sign can be explained. In both cases, free energy must decrease. In the first case, this is achieved by reducing its enthalpic and entropic components with $|\Delta H|>|T \Delta s|$ when the momentums $P_{i n}$ received from the photons emitted into the region between the objects are less than the momentums $P_{\text {out }}$ from the photons emitted to the outer region, i.e. $P_{\text {in }}<P_{\text {out }}$. In the second case, the enthalpic and entropic components increase. At the same time $|\Delta H|<|T \Delta s|$ and $P_{\text {in }}>P_{\text {out }}$.

One of the main contributors to the interaction of objects at a large distance is made by electromagnetic radiation. It may seem that it is not enough to significantly affect the dynamics of the behavior of objects. Indeed, the photon energy of infrared radiation with a frequency of $1 \mathrm{THz}$ is equal to $E=h v \approx 6.6 \times 10^{-22} \mathrm{~J}$. But an amount of light more typical in everyday experience is the energy equivalent to that of one mole of photons. Its energy can be calculated by multiplying the photon energy by the Avogadro constant, $N_{\mathrm{A}} \approx$ $6.022140758(62) \times 10^{23} \mathrm{~mol}^{-1}$, with the result of $E \approx 0.4 \mathrm{~kJ} / \mathrm{mol}$, about the scale adapted to humans, where energies are typical of the order of $E \approx 1 \mathrm{~kJ}$. It is clear that macroscopic objects radiate significantly larger energy being mutually produced by their different "charges".

Any object radiates the heat if its temperature is higher than the temperature of the environment. According to Fourier's law of the thermal conduction, the local heat flux is proportional to the negative local temperature gradient:

$$
q=-k_{1} \Delta T
$$

where $q$ is the local heat flux density, $k_{1}$ is the material's heat conductivity and $\Delta T$ is the temperature gradient.

Local areas where the "Big Bangs" took place can be considered as objects 
with described entropy interaction between themselves. Universes formed in such areas must through entropic interactions run away or entropically repel each other, thus increasing the entropy of the World space, which has become structured. This "divergence" of Universes excludes their meeting and interpenetration.

\section{Conclusions}

Consideration of the presence of virtual objects in the World space, a possible explanation of their nature and the ability to transfer into the real state, appearance of objects with an electric charge proved using the Principle of Least Action and Le Chatelier's Principle allow us to achieve a better understanding of the Universe emergence and evolution process. The supplement of the entropic interaction (leading to bifurcations) to the current cosmological theory provides an opportunity to utilize thermodynamic laws to explain these processes. "Corpuscular-Field dualism" makes it possible to obtain a detailed description of such processes. All of these developments are not negate any laws of Nature using in theoretical physics.

The considered realization of the entropy interaction of material objects by means of using their emission of energy quanta is common to all fundamental interactions and can serve as the basis for building a unified field theory.

The possible infinitely large number of Universes guarantees, with a non-zero probability, the presence of various Universes with any set of world constants that determine the laws of Nature. In one of these Universes, a set of world constants turned out to be suitable for the emergence and further evolution of organic life as happened on our planet Earth and progressed into the animated world we live in today. Therefore, we cannot agree with the Anthropic Principle [46] that argues that Nature specially selected a set of world constants for our Universe in such a way that they are acceptable to the existence of humanity.

Although the set of world constants that determine the laws of Nature are acceptable to the existence of humanity, the living organisms on our Planet had to be significantly adjusted to survive. The use of the thermodynamic approach allowed Jeremi England with colleagues to develop an alternative of the Darwin theory of evolution. In their works [47] it is shown that the more rapid growth of entropy of living organisms can be the defining factor of the survival of biological objects in their competitive fight for existence. This result is coordinated very well with the idea stated by E. Schrödinger: "Entropy at a quicker rate affords a more intense life process". According to Schrödinger, in a living organism continuously there is "a new order", thanks to consumption of "some order" from the environment. In other words, the concept of "order from order" works here. It was suggested by Schrödinger in 1944 on the basis of thermodynamics in his book "What is Life?" [48].

\section{Conflicts of Interest}

The authors declare no conflicts of interest regarding the publication of this paper. 


\section{References}

[1] Friedmann, A. (1922) Über die Krümmung des Raumes. Zeitschrift für Physik, 10, 377-386. https://doi.org/10.1007/BF01332580

[2] Lemaître, G. (1927) Un Univers homogène de masse constante et de rayon croissant rendant compte de la vitesse radiale des nébuleuses extra-galacticos. Annales de la Société Scientifique de Bruxelles, A47, 49-59.

[3] Hubble, E. (1926) Extragalactic Nebulae. The Astrophysical Journal, 64, 321-369. https://doi.org/10.1086/143018

[4] Hubble, E. (1929) A Relation between Distance and Radial Velocity among Extra-Galactic Nebulae. Proceedings of the National Academy of Sciences, 15, 168-173. https://doi.org/10.1073/pnas.15.3.168

[5] De Sitter, W. (1930) On the Magnitudes, Diameters, and Distances of the Extragalactic Nebulae and Their Apparent Radial Velocities. Bulletin of the Astronomical Institutes of the Netherlands, 5, 157.

[6] Einstein, A. and De Sitter, W. (1932) On the Relation between the Expansion and the Mean Density of the Universe. Proceedings of the National Academy of Sciences, 18, 213-214. https://doi.org/10.1073/pnas.18.3.213

[7] Hawking, S. and Rayner, G. (1973) The Large Scale Structure of Space-Time. Cambridge University Press, Cambridge. https://doi.org/10.1017/CBO9780511524646

[8] Guth, A. (1997) The Inflationary Universe. Perseus Books, Reading.

[9] Starobinskii, A. (1979) Spectrum of Relict Gravitational Radiation and the Early State of the Universe. Pis' ma $v$ Zhurnal Éksperimental noi $i$ Teoreticheskoi Fiziki, 30, 719-723.

[10] Linde, A. (1982) A New Inflationary Universe Scenario: A Possible Solution of the Horizon, Flatness, Homogeneity, Isotropy and Primordial Monopole Problems. Physics Letters B, 108, 389-393. https://doi.org/10.1016/0370-2693(82)91219-9

[11] Vilenkin, A. (2015) The Beginning of the Universe. Inference: International Review of Science 1/4.

[12] Bresler, S. and Erusalimsky, B. (1965) Physics and Chemistry of Macromolecules. Nauka, Moscow.

[13] Volkenstein, M.V. (1959) Configuration Statistics of Polymeric Chains. Prod. Academy of Sciences of the USSR.

[14] Vilenchik, L. (2016) Quintessence: A Thermodynamic Approach to the Phenomena of Nature. Nova Science Publishers, Hauppauge.

[15] Vilenchik, L. (2017) Entropic Essence of Nature. International Journal of Theoretical Physics, 17, 295-307.

[16] Vilenchik, L. (2018) Coexistence of Short-Range and Long-Range Actions at Interactions of Material Objects and Phase Transitions. Journal of Nature Science and Sustainable Technology, 12, 131-139.

[17] Verlinde, E. (2011) On the Origin of Gravity and the Laws of Newton. Journal of High Energy Physics, 4, 29. https://doi.org/10.1007/JHEP04(2011)029

[18] Taylor, P. and Tabachnik, J. (2013) Entropic Forces-Making the Connection between Mechanics and Thermodynamics in an Exactly Soluble Model. European Journal of Physics, 34, 729-736. https://doi.org/10.1088/0143-0807/34/3/729

[19] Landau, L. (1937) On the Theory of Phase Transitions. Zhurnal Eksperimentalnoi $i$ Teoreticheskoi Fiziki, 7, 19-32.

[20] Slater, J.C. (1951) A Simplification of the Hartree-Fock Method. Physical Review, 
81, 385. https://doi.org/10.1103/PhysRev.81.385

[21] Hensen, B., et al. (2015) Loophole-Free Bell Inequality Violation Using Electron Spins Separated by 1.3 Kilometers. Nature, 526, 682-686.

https://doi.org/10.1038/nature15759

[22] Bell, J. (1964) On the Einstein Podolsky-Rosen-Paradox. Physics, 1, 195-200. https://doi.org/10.1103/PhysicsPhysiqueFizika.1.195

[23] Pierre, A. (2016) On the Applicability of Bell's Inequality. Progress in Physics, 12, 211-215.

[24] Prigogine, I. (1997) The End of Certainty. Time, Chaos, and New Laws of Nature. The Free Press. New York.

[25] Prigogine, I. and Stengers, I. (1984) Order Out of Chaos. University of Michigan, Bantam Books, Ann Arbor.

[26] Mach, E. (1909) Mechanics.

[27] Vilenchik, L. (2017) Psychological Treatment as a Manifestation of Thermodynamic Principles. International Journal of Psychology Research, 11, 125-132.

[28] Vilenchik, L. and Vilenchik, M. (2019) Entropic Change Is a Ruler of the Biological Rhythms and Time. Journal of Nature Science and Sustainable Technology, 13, 47-55.

[29] Einstein, A. (1916) The Foundation of the General Theory of Relativity. Annalen der Physik, 49, 769-822. https://doi.org/10.1002/andp.19163540702

[30] Carroll, S. (2007) Dark Matter, Dark Energy: The Dark Side of the Universe. The Teaching Company, Chantilly, Guidebook Part 2, 46.

[31] Belenky, B. and Vilenchik, L. (1983) Modern Liquid Chromatography of Macromolecules. Elsevier, Amsterdam.

[32] Greiner, W. and Müller, B. (2000) Gauge Theory of Weak Interactions. Springer, Berlin. https://doi.org/10.1007/978-3-662-04211-3

[33] Kossmann-Schwarzbach, Y. (2010) The Noether Theorems: Invariance and Conservation Laws in the Twentieth Century. Sources and Studies in the History of Mathematics and Physical Sciences. Springer-Verlag, Berlin. https://doi.org/10.1007/978-0-387-87868-3_3

[34] Prigogine, I. (1955) Introduction to Thermodynamics of Irreversible Processes. Charles C. Thomas Publisher, Springfield.

[35] de Maupertuis, P.L.M. (1744) Accord de différentes lois de la nature qui avaient jusqu'ici paru incompatibles. Mém. As. Sc., Paris, 417.

[36] García-Morales, V., Pellicer, J. and Manzanares, J. (2008) Thermodynamics Based on the Principle of Least Abbreviated Action: Entropy Production in a Network of Coupled Oscillators. Annals of Physics, 323, 1844-1858. https://doi.org/10.1016/j.aop.2008.04.007

[37] Feynman, R. (1942) The Principle of Least Action in Quantum Mechanics. PhD Dissertation, Princeton University, Princeton.

[38] Kline, M. (1972) Mathematical Thought from Ancient to Modern Times. Oxford University Press, New York, 167-168.

[39] Elsgolts, L. (1961) Differential Equations, and Calculus Variations. Paramount Press, Panama, 389-399.

[40] Linde, A. (1990) Particle Physics and Inflationary Cosmology. Harwood, Chur. https://doi.org/10.1201/b16971

[41] Knox, K. (1985) Le Chatelier's Principle. Journal of Chemical Education, 62, 863. 
https://doi.org/10.1021/ed062p863

[42] Dirac, P. (1928) The Quantum Theory of the Electron. Proceedings of the Royal Society $A, 117,610$.

[43] Bohr, N. (1928) The Quantum Postulate and the Recent Development of Atomic Theory. Nature, 121, 580-590. https://doi.org/10.1038/121580a0

[44] Einstein, A. (1907) On the Principle of Relativity and the Consequences Derived from It. Yearbook of Radioactivity and Electronics 4.

[45] Mössbauer, R. (1958) Nuclear Resonance Fluorescence of Gamma Radiation in Ir191. Journal of Physics, 151, 124-143. https://doi.org/10.1007/BF01344210

[46] Carter, B. (1974) Large Number Coincidences and the Anthropic Principle in Cosmology. In: IAU Symposium 63: Confrontation of Cosmological Theories with $\mathrm{Ob}$ servational Data, Riedel, Dordrecht, 291-298.

https://doi.org/10.1007/978-94-010-2220-0_25

[47] England, J. (2013) Statistical Physics of Self-Replication. The Journal of Chemical Physics, 139, Article ID: 121923. https://doi.org/10.1063/1.4818538

[48] Schrodinger, E. (1992) What Is Life. Cambridge University Press, Cambridge. https://doi.org/10.1017/CBO9781139644129 